memory is very scarce at the moment. Its speed on a Sun or Macintosh II is more than adequate, but the version for Macintosh Plus or SE is likely to be frustratingly slow. As with any new software Mathematica is not without bugs, so upgrades are essential; these are free for 90 days, but will require a (modest) continuing investment thereafter.

These caveats aside, Mathematica is a startlingly good tool. Research groups with Suns or Macintosh IIs should acquire it. Indeed, the program is, by itself, nearly enough justification to buy the necessary hardware. There is no reason in principle that it cannot also be made available for VAX workstations and 386-based MSDOS (but not 8086 or 286) machines; I hope that happens soon.

William H. Press is at the Harvard College Observatory, Harvard University, Cambridge, Massachusetts 02138, USA.

\section{Moving story}

Roger Lemon

The Neural and Behavioural Organization of Goal-Directed Movements. By Marc Jeannerod. Clarendon: 1988. Pp.283. $£ 32.50, \$ 49.95$.

C. S. Sherrington wrote that "To move things is all mankind can do; for such the sole executant is muscle, whether in whispering. . . or in felling a forest". Understanding the organization of movements remains a central issue in research on brain and behaviour. And, by common consent, attempts to do so require theoretical and experimental studies of a truly multidisciplinary character.

Marc Jeannerod's book is an excellent example of what can be achieved in this way. Its publication marks a point when the many approaches to the problem of understanding movement are rapidly converging. Among them is the use of inverse kinematics to predict the way in which a movement is initiated and controlled by studying its dynamic features. Jeannerod also draws on experimental evidence from two other fields: the neurophysiology of behaving animals, in which neuronal activity in motor areas of the brain is monitored during movement; and the use of cognitive psychology to study motor control in human subjects. Jeannerod examines how these approaches have furthered our knowledge of the organization of goal-directed movements. A good example is the visual guidance of the hand towards a specified target. Jeannerod has made notable contributions in this area, and the best parts of his book are those in which he uses this type of movement to illustrate some basic principles of motor organization.

The book opens with a consideration of the main theoretical arguments for a central representation of motor programs'. As Jeannerod writes, "Movement is not a transparent phenomenon", thereby neatly stating a central problem for those striving to understand movement and its control by the brain. When compared with the working of our sensorium, we have relatively little insight or perception of how we produce and control movements. Ideas about how the brain organizes movement have a long and interesting background, well discussed in Jeannerod's earlier book, The Brain Machine. Here he shows how hierarchical theories of neural organization have provided the main theoretical framework for investigation of the motor system. In the 'top-down' model of motor representation, Marr proposed a hierarchy consisting of a highest level of 'computational theory' which defines the goal of the computation; an intermediary level which transforms the computed input to an output; and finally a third, hardware level for implementing the output. But we now know that even the most elementary motor processes can be influenced by specific cognitive states or behavioural 'set', and this makes the identification and separation of the different hierarchical levels extremely difficult.

Jeannerod is an excellent guide to this difficult area. His account is readable and full of direct experimental evidence for a hierarchical organization. In visuo-motor reaching, for instance, there is evidence that the central command for moving the eye, head and hand occurs simultaneously; the resulting movements have successively later onsets largely because the mechanical properties of these different segments are so different. Earlier authors have not shrunk from including, without much justification, the term 'control' in the titles of their books. Here, Jeannerod explicitly attacks the question of what it is that is being controlled, his examples including the direction and amplitude of movements and the co-ordination of the proximal (arm) and distal (hand) components during reaching. This coordination, although very precise, is controlled by different neural mechanisms.

Although knowledge of the neural machinery that underpins movement has resulted mainly from animal studies, our understanding of how this neural machinery is put to work before and during voluntary movement has come increasingly from experiments in human beings. These studies have combined ingenious design with new techniques for registering movement. Jeannerod's approach has been radically different to that of much of the work on animals, where there has been a tendency to "reduce complex movements to units functioning in the same mode as single joint movements". In a strong plea for more research into multi-segmental actions, such as eye-hand control, he points out that "parameters currently considered in the study of single-joint movements, such as latency, duration, error with respect to the target and the kinematics of the end point of the limb are far from representing an adequate description of motor performance". Investigations of complex movements show that their trajectory is somehow abstracted from the movements at individual joints, and these studies have revitalized the quest for the neural mechanisms which can perform such an abstraction. In this regard it is remarkable that there is no mention in the book of the work of Georgopoulos and his colleagues on the activity of monkey cortical neurons during reaching.

In some of the most controversial areas Jeannerod is refreshingly non-partisan, preferring to present the available evidence in a logical sequence. The theoretical territory of many areas of motor control has all too often been staked out by the protagonists of mutually exclusive theories, and experimental results have been manipulated to fit one or other of these theories. It has long been argued, for example, that feedback control of movement depends either on the afferent information arising in peripheral receptors, or on internal feedback/corollary discharge mechanisms, by which the brain is constantly informed of movement by monitoring its own command signals. But sensory and internal feedback mechanisms cannot be considered to be mutually exclusive, because the former provides the rules by which the latter operates once a particular movement has been learned. As an example, Jeannerod reviews the evidence showing that disruption of sensory feedback from the eye muscles produces errors in reaching for visible targets. On a separate line of reasoning, he shows that the maps of proprioceptive and visual modalities must remain in register with one another in order for them to interact for successful reaching into extrapersonal space. In the last chapter he discusses the pivotal role of the posterior parietal cortex in co-ordinating poly-sensory inputs for the control of reaching.

Although this book will be of particular interest to those studying visuo-motor behaviour, it deserves to reach a much wider audience. It has a clarity of style and of argument that makes it a rewarding read for all those interested in understanding how the brain organizes such tasks as forest-felling and whispering.

Roger Lemon is in the Department of Anatomy, University of Cambridge, Downing Street Cambridge CB2 3DY, UK. 